\title{
Numerical simulation of multi-layer 3D concrete printing
}

\author{
Jon Spangenberg ${ }^{1 *}$, Wilson Ricardo Leal da Silva ${ }^{2}$, Raphaël Comminal ${ }^{1}$, Md. Tusher Mollah ${ }^{1}$, \\ Thomas Juul Andersen ${ }^{2}$, Henrik Stang ${ }^{3}$
}

\footnotetext{
${ }^{1}$ Department of Mechanical Engineering, Technical University of Denmark, Kgs. Lyngby, Denmark

${ }^{2}$ Danish Technological Institute, Taastrup, Denmark

${ }^{3}$ Department of Civil Engineering, Technical University of Denmark, Kgs. Lyngby, Denmark
}

Received: 16 April 2021 / Accepted: 14 September 2021 / Published online: 08 October 2021

(C) The Author(s) 2021. This article is published with open access and licensed under a Creative Commons Attribution 4.0 International License.

\begin{abstract}
This paper presents a computational fluid dynamics model fit for multi-layer 3D Concrete Printing. The numerical model utilizes an elasto-visco-plastic constitutive model to mimic the flow behaviour of the cementitious material. To validate the model, simulation data is compared to experimental data from 3D printed walls. The obtained results show that the numerical model can reproduce the experimental results with a high accuracy and quantify the extrusion load imposed upon the layers. Such load is found to exceed the material's yields stress in certain regions of previously printed layers, leading to layer deformation/flow. The developed and validated numerical model can assist in identifying optimal printing strategies, reducing the number of costly experimental print failures and human-process interaction. By doing so, the findings of this paper help 3D Concrete Printing move a step closer to a truly digital fabrication process.
\end{abstract}

Keywords: 3D concrete printing; Computational fluid dynamics; Multi-layer prints; Rheology

\section{Introduction}

3D Concrete Printing (3DCP) is a construction technology that currently receives a lot of attention both from academia and industry, since it comes with the promise of reducing cost and production time of concrete structures [1,2]. 3DCP is an umbrella term covering several digital fabrication methodologies. The most applied technique is extrusionbased 3DCP [3], which is the focus of this study. The 3DPC process steps are adapted from material extrusion additive manufacturing of polymers [4], where the printer tool path is generated by a slicing software in the form of a G-code, which is based on a STL file that constitutes a virtual 3D representation of the structure.

Extrusion-based 3DCP has been applied successfully by the industry to produce pre-cast elements, bridges, and non-loadcarrying walls for houses. However, the technology faces barriers such as the (currently) inexistence of local building regulations and technical standardization fit for 3DCP, let alone the need to improve process robustness [5]. In addition, when printing new materials or geometries, the deployment of 3DCP is still dependent on extensive (and costly) trial-anderror procedures, which substantially limits the fabrication method in terms of agile and autonomous production. This barrier needs to be overcome to unleash extrusion-based 3DCP's potential as a digital fabrication technology.
In the researchers' quest to understand and improve extrusion-based 3DCP, they recently develop various numerical models that simulates the process [6]. For example, Wolfs et al. $[7,8]$ used finite element models to investigate the early age mechanical behaviour of printed concrete as well as the structural collapse during the material extrusion printing process. Nguyen-Van et al. [9] applied a 3D nonlinear quasistatic FE model to obtain thorough insight into the 3DCP process. Reinold et al. [10,11] carried out particle finite element simulations of the fresh cementitious material during 3DCP. Recently, Comminal et al. $[12,13]$ compared the cross-sectional shape of single layer 3DCP experiments with predictions from a computational fluid dynamics (CFD) model simulating the process - this was an attempt to understand the influence of 3DCP processing parameters on the layer geometry. The latter study showed a good agreement between the numerical and experimental results when applying an elasto-visco-plastic material model in the CFD model.

This paper aims at further validating the developed CFD model with appurtenant elasto-visco-plastic constitutive material behaviour by comparing its results to cross-sections of experimental multi-layer prints. Furthermore, the paper aims to exploit the numerical model in order to obtain previously unknown knowledge on the inter-layer mechanical behaviour of the fresh concrete during deposition.

\footnotetext{
* Corresponding author: Jon Spangenberg, E-mail: josp@mek.dtu.dk
} 


\section{Experiments and simulation}

The multi-layer prints were carried out with a mortar that consisted of White Cement (CEM I 52.5 R - SR 5 (EA)), limestone filler, water, and admixtures. The filler-to-cement ratio was 0.82 , and the water-to-cement ratio was 0.39 . The admixtures included a high-range water reducer admixture ( $0.1 \%$ by weight of cement - bwoc.), a viscosity-modifying agent ( $0.1 \%$ bwoc.), and a retarder admixture ( $0.5 \%$ bwoc.). The latter facilitated a rheological time independent mortar during deposition of the multiple layers, decreasing the complexity of the material model adapted by the CFD model.

The mortar had a density of $2100 \mathrm{~kg} / \mathrm{m}^{3}$ and was mixed in an Eirich Intensive Mixer Type R08W with a multiple step procedure. The rheological characterization of the mortar was performed on an Anton Paar MCR 502 rheometer using a vane-in-cup measuring device. By rotational tests, a yield stress $\tau_{0}=630 \mathrm{~Pa}$ and plastic viscosity $\mu_{p}=7.5$ Pa.s were determined. In addition, oscillatory tests were performed to determine the storage modulus $G^{\prime}=200$ $\mathrm{kPa}$ in the material's linear visco-elastic region, which was used as the linear-elastic shear modulus of the unyielded material. For detailed information on the material, mixing procedure, and characterization protocol, please refer to [13]. The experimental multi-layer prints were produced with the 3DCP setup seen in Fig. 1a. The setup consists of a 6 -axis robot arm, a pump equipped with a hopper, a steel-wire rubber hose, and a custom 3D printed polymer nozzle, cf. [14] for detailed information. The 3DCP setup was used to print small walls comprising from one to three layers. The extrusion nozzle had a diameter $D=25 \mathrm{~mm}$, and the deposition was carried out with a print speed $V=30 \mathrm{~mm} / \mathrm{s}$ and extrusion rate $U=33.6 \mathrm{~mm} / \mathrm{s}$. The distance between the nozzle and substrate (build plate) was set at $12.5 \mathrm{~mm}$ for the first layer, and incrementally increased with the same height for the subsequent layers. After the mortar hardened, four crosssectional cuts were scanned for each of the three walls to quantify their cross-sectional shapes, see example in Fig. 1 b. The digitalization of the cross-sections was obtained by a custom image processing script outlined in [13].

The numerical model was developed in the commercial CFD software, $F L O W-3 D^{\circ}$. The software has successfully simulated the benchmark test for flow of fresh cementitious materials [15] as well as other concrete phenomena such as flow in a VHPC-precambered composite beam [16], and aggregate segregation in self-compacting concrete [17]. The CFD model developed for simulating the multi-layer printing in this study is depicted in Fig. 2. The model applied the finite volume method to discretize the governing equations (i.e., the mass and momentum conservation equations) and a semi-implicit iterative solver was used to find the pressure- and velocityfields. In addition, the volume of fluid method [18] facilitated the tracking of the free surface of the cementitious mortar, while the immersed boundary method enabled the representation and movement of the nozzle within the computational domain. The boundary condition on the nozzle and substrate was a no-slip condition, and the model exploited the symmetry of the problem to reduce the computational effort. The mortar was simulated with the elasto-visco-plastic material model described in [13].

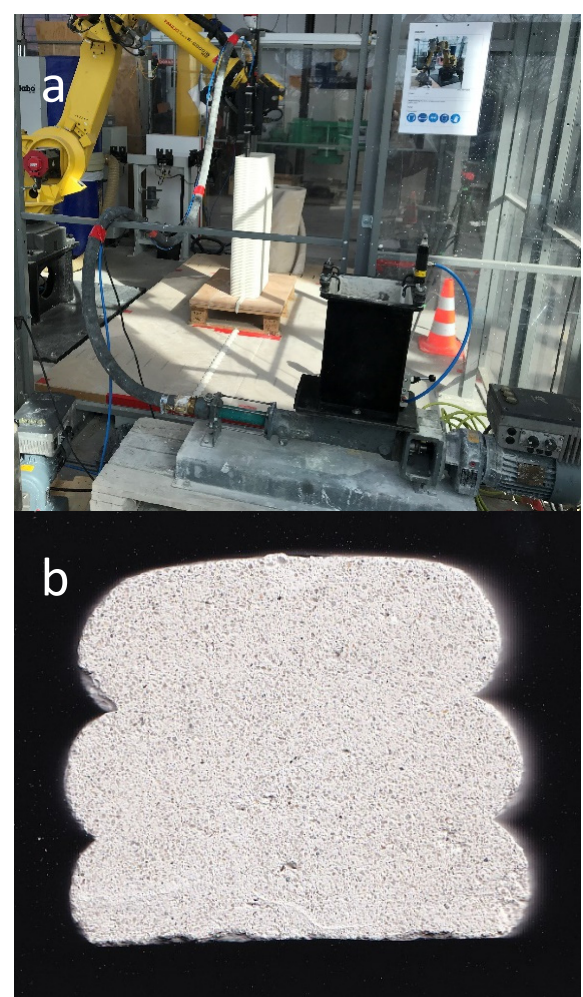

Figure 1. a) 3DCP setup and b) cross-sectional shape of a multi-layer print.

The simulated rheological properties were equal to the values determined experimentally, with the only exception that the linear-elastic shear modulus was set at $100 \mathrm{kPa}$ instead 200 $\mathrm{kPa}$ to mitigate numerical instabilities arising from the unyielded part of the material. This change is not expected to critically alter the simulation outcome (as later discussed in the results' section), but rather a numerical solution to inexpensively soften the non-linear behaviour of the elastovisco-plastic material, which is notoriously difficult to handle numerically. All modelled process parameters (i.e., printing speed, extrusion rate, and nozzle height) were adapted from the real 3DCP experiment.

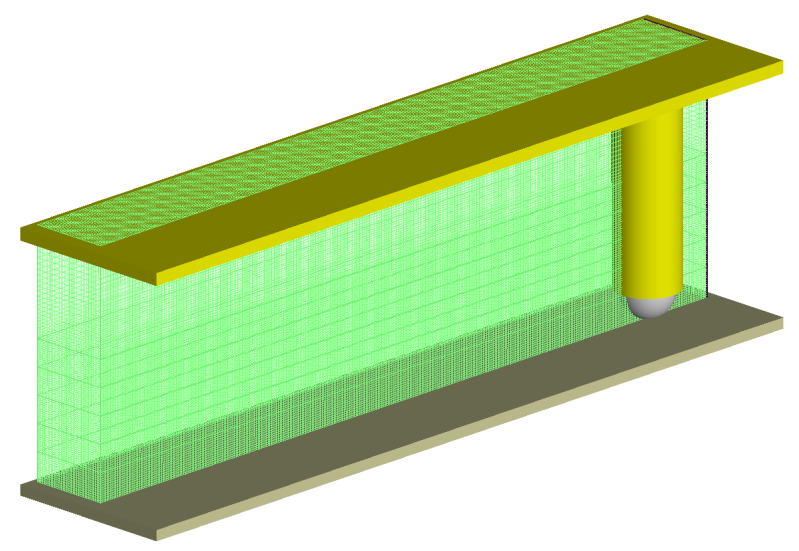

Figure 2. Computational domain for the CFD model. 


\section{Results and Discussion}

A snapshot of the CFD model simulating the multi-layer printing process is shown in Fig. 3. Since the mortar is simulated as an elasto-visco-plastic material with a relatively high yield stress, the first layer can withstand the pressure of the subsequent layers without collapsing. The model also predicts a grooved surface on the side walls coming from the individual mortar layers, which is commonly observed in the 3DPC process when using a circular nozzle.

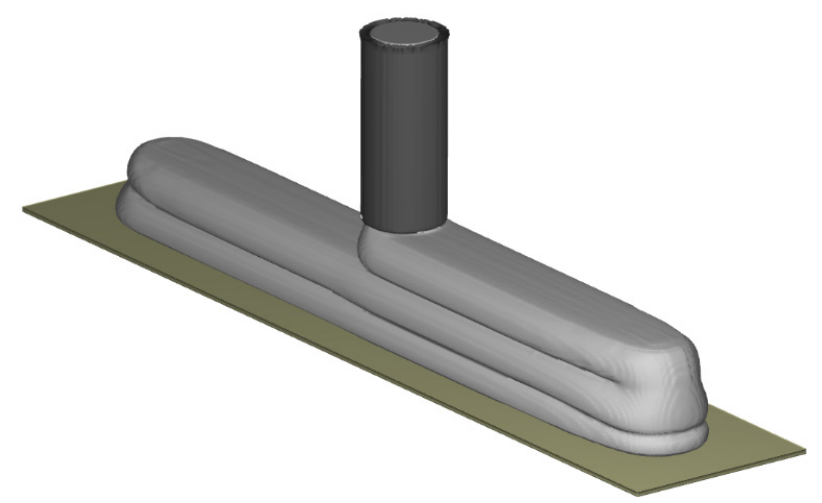

Figure 3. CFD model simulating multi-layer 3DCP.

In Fig. 4, the cross-sectional shapes (after each layer deposition) are plotted for both the CFD model and 3DCP experiment to carry out a quantitative comparison. The simulated cross-sectional shape is obtained by a cutting plane in the middle of the model, while the experimental crosssections originate from the four cuts of the hardened printed walls.

Overall, the numerical and experimental results agree well, indicating that the selected CFD model is capable of mimicking the 3DCP process accurately and that the reduction of the linear-elastic shear modules to $100 \mathrm{kPa}$ is not detrimental for the simulations precision. Specifically, the model accurately predicts the width and height of the three layers as well as the grooved surface on the side walls. The only inconsequential discrepancy observed in the results is after the deposition of the second layer, where the model slightly underestimates the cross-section width. However, given the inevitable uncertainty related to any experiment, the observed discrepancy can be accepted. In this experiment, the width of the bottom layer is a bit larger in the wall with two-layers as compared to the wall with threelayers, which might be attributed to minor variation in the process parameters during printing.

Moreover, the results in Fig. 4 show that the width of a previously printed layer increases when depositing a new layer on top. This illustrates that the pressure applied by the new material during extrusion exceeds the yield stress of the material on which it is printed upon.
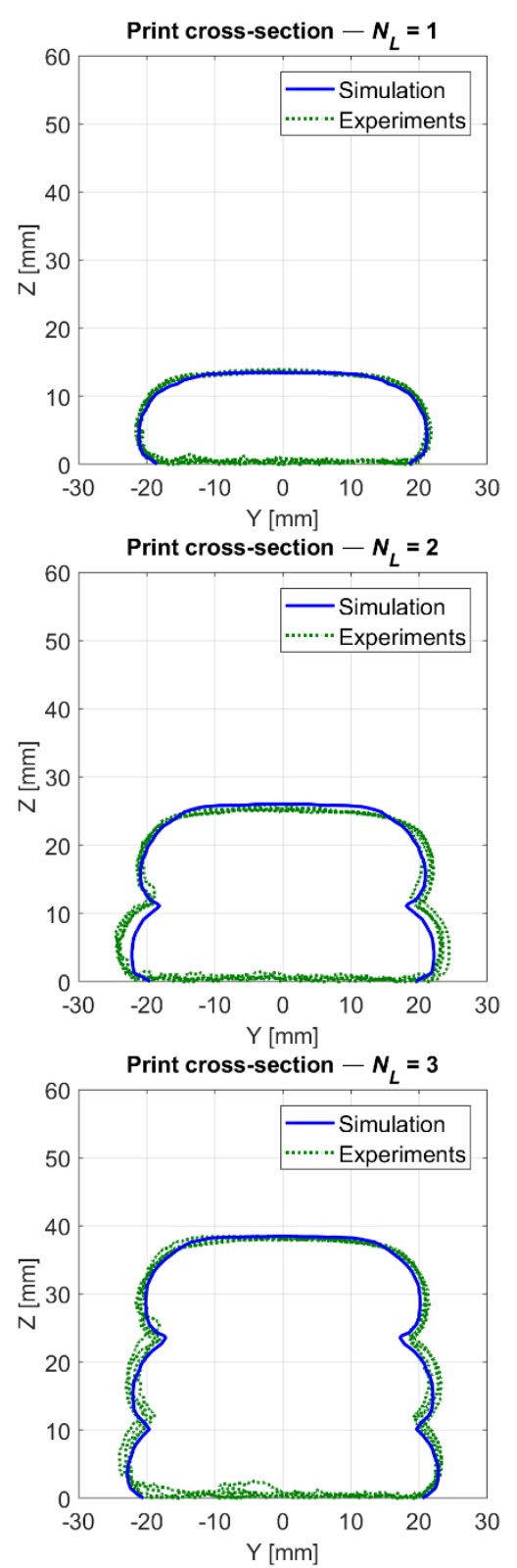

Figure 4. Cross-sectional shapes obtained from CFD model and 3DCP experiments.

The CFD model provides a virtual window into the process, which allows for quantifying the yielded and unyielded zones of the material during deposition (Fig. 5). The figure indicates that, while printing the third layer, a part of the second layer is yielded (green zone immediately below the nozzle), leading to deformation/flow and thus, an increase in the width of the second layer. This interaction between the two layers, combined with a sufficiently short interlayer interval time, is likely a complementary reason for researchers reporting a good interlayer strength in 3DCP [19]. The figure also shows that the entire first layer stays unyielded and does not change its width. 


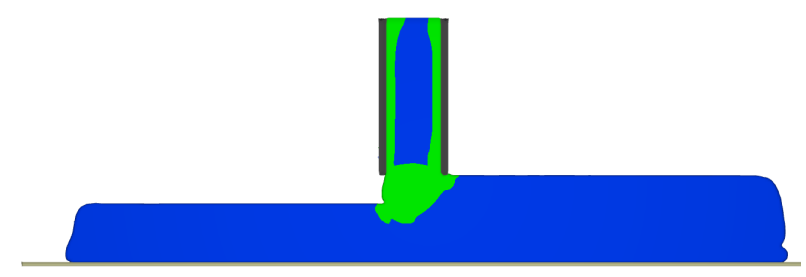

Figure 5. Simulation results of yielded (green) and unyielded (blue) material during deposition of the third layer. The view is a cross-sectional cut parallel to the deposition direction in the middle of the nozzle.

In Fig. 6, the pressure (P), vertical deviatoric component of the elastic stress tensor $\left(E_{z z}\right)$, and total extrusion load $\left(P+E_{z z}\right)$ are plotted for each deposition step. These three components are numerically found at the nozzle outlet. In this figure, it can be noticed that the total extrusion load exceeds the mortar's yield stress of $630 \mathrm{~Pa}$ and initiates flow. In addition, the total extrusion load is higher for the first layer as compared to the subsequent layers, because the first layer is printed on a solid substrate (build plate), whereas the next layers are deposited on the deformable mortar. This means that, to achieve a geometrically stable print (i.e., consecutive layers with the same height and width), one must ensure that the deformation pattern of previously printed layers are the same. Such task is not trivial for a time-dependent material, since this puts considerable restrictions on when new layers can be deposited. Alternatively, the structural build-up of the mortar must be so quick that it can withstand the total extrusion load of the next layers, but this can be a challenge in terms of reduced interlayer strength or clogging of the 3DCP system. In this regard, the presented CFD model can be used as a tool that helps identify optimal printing strategies, which lead to stable prints for mortars with varying rheological response.

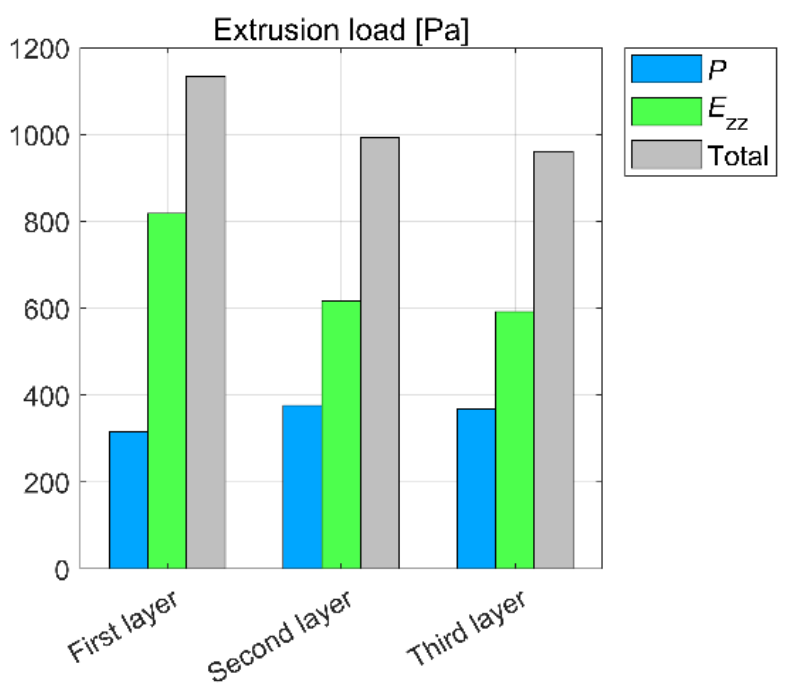

Figure 6. Simulation results of the pressure $(P)$, vertical deviatoric component of the elastic stress tensor $\left(E_{z z}\right)$, and total extrusion load during deposition of three layers.

\section{Conclusion and research prospects}

This work presented experimental data and CFD model results of multi-layer 3DCP. The results were in good agreement and can be considered as an extended validation of the CFD model that utilizes an elasto-visco-plastic material behaviour, first introduced in [13]. Furthermore, the model predicted the yielded and unyielded parts of the mortar during deposition, and it illustrated that the total extrusion load while printing a layer would exceed the yield stress of the layer underneath, which led to flow/deformation. Consequently, the model can become extremely useful when trying to find tool paths and printing process parameters that lead to stable prints when printing new materials or geometries.

The drawback of the CFD model is that it takes several days to complete one simulation, limiting the models applicability in an industrial setting and making it virtually impossible to couple with a 3DCP system in a closed loop. Particularly, the elastic part of the constitutive law contributed to the model being computational expensive as well as numerical difficult to handle. In fact, the model failed to simulate a five-layers print due to numerical instabilities. Therefore, future research will involve finding a computation methodology that makes the model numerically more robust.

Though the CFD model is inherently computational expensive, it is well-suited for running parametric studies, since several scenarios can be executed in parallel. As such, the model serves as a basis for reducing the number of experiments to run in the lab or field, e.g. enabling an easy and cost-effective way to determine optimal printing strategies for the material flow around rebars - that being an open research question at present.

\section{Acknowledgement}

The authors would like to acknowledge the support of the Innovation Fund Denmark (Grant no. 8055-00030B: Next Generation of 3D-printed Concrete Structures), as well as the support of COWIfonden. Moreover, the authors thank FLOW$3 D$ for their support in regards to licenses.

\section{CRediT authorship statement}

Jon Spangenberg: Conceptualization, Methodology, Formal analysis, Supervision, Writing - original draft.

Wilson Ricardo Leal da Silva: Conceptualization, Methodology, Investigation, Writing - original draft, Funding acquisition.

Raphaël Comminal: Conceptualization, Methodology, Formal analysis, Investigation, Visualization, Writing - review and editing.

Md. Tusher Mollah: Formal analysis, Investigation, Writing review and editing.

Thomas Juul Andersen: Supervision, Project administration, Funding acquisition.

Henrik Stang: Supervision, Project administration, Funding acquisition. 


\section{References}

[1] S. C. Paul, G. P. A. G. van Zijl, M. J. Tan, I. Gibson, A review of 3D concrete printing systems and materials properties: current status and future research prospects. Rapid Prototyp J (2018) 24: 784-798, https://doi.org/10.1108/RPJ-09-2016-0154.

[2] V. Mechtcherine, V. N. Nerella, F. Will, M. Näther, J. Otto, M. Krause, Large-scale digital concrete construction-CONPrint3D concept for on-site, monolithic 3D- printing. Automat Constr (2019) 107: 102933. https://doi.org/10.1016/i.autcon. 2019.102933.

[3] R. A. Buswell, W. R. Leal da Silva, F. P. Bos, H. R. Schipper, D. Lowke, N. Hack, H. Kloft, V. Mechtcherine, T. Wangler, N. Roussel, A process classification framework for defining and describing Digital Fabrication with Concrete. Cem Concr Res (2020) 134: 106068. https://doi.org/10.1016/j.cemconres.2020.106068

[4] M. P. Serdeczny, R. Comminal, D. B. Pedersen, J. Spangenberg, Experimental and analytical study of the polymer melt flow through the hot-end in material extrusion additive manufacturing. Addit Manuf (2020) 32: 100997.

https://doi.org/10.1016/i.addma.2019.100997

[5] R. A. Buswell, W. R. Leal da Silva, S. Z. Jones, J. Dirrenberger, 3D printing using concrete extrusion: a roadmap for research, Cem Concr Res (2018) 112: 37-49.

https://doi.org/10.1016/i.cemconres.2018.05.006.

[6] N. Roussel, J. Spangenberg, J. Wallevik, R. J. M. Wolfs, Numerical simulations of concrete processing: From standard formative casting to additive manufacturing, Cem Concr Res (2020) 135: 106075. https://doi.org/10.1016/i.cemconres.2020.106075

[7] R. J. M. Wolfs, F. P. Bos, T. A. M. Salet, Early age mechanical behaviour of $3 \mathrm{D}$ printed concrete: numerical modelling and experimental testing. Cem Concr Res 106 (2018) 103-116, https://doi.org/10.1016/i.cemconres.2018.02.001.

[8] R. J. M. Wolfs, A. S. J. Suiker, Structural failure during extrusion-based 3D printing processes. Int. J Adv Manuf Tech (2019) 1-20, https://doi.org/10.1007/s00170-019-03844-6.

[9] V. Nguyen-Van, B. Panda, G. Zhang, H. Nguyen-Xuan, P. Tran, Digital design computing and modelling for 3-D concrete printing. Automat Constr (2021) 123: 103529. https://doi.org/10.1016/i.autcon.2020.103529

[10] J. Reinold, V. N. Nerella, V. Mechtcherine, G. Meschke, Particle finite element simulation of extrusion processes of fresh concrete during 3D-concrete-printing, II International Conference on Simulation for Additive Manufacturing, Pavia, Italy, September 11-13, 2019, 428439.

[11] J. Reinold, G. Meschke, Particle finite element simulation of fresh cement paste - inspired by additive manufacturing techniques, Proc Appl Math Mech (2019) 19: e201900198. https://doi.org/10.1002/pamm.201900198

[12] [12] R. Comminal R., W. R. Leal da Silva, T. J. Andersen, H. Stang, J. Spangenberg, Influence of Processing Parameters on the Layer Geometry in 3D Concrete Printing: Experiments and Modelling. In: Bos F., Lucas S., Wolfs R., Salet T. (eds) Second RILEM International Conference on Concrete and Digital Fabrication. DC 2020. RILEM Bookseries, vol 28. Springer, Cham. (2020). https://doi.org/10.1007/978-3-030-49916-7 83

[13] R. Comminal, W. R. Leal da Silva, T. J. Andersen, H. Stang, J. Spangenberg, Modelling of 3D concrete printing based on computational fluid dynamics, Cem Concr Res (2020) 138: 106256. https://doi.org/10.1016/i.cemconres.2020.106256

[14] W. R. Leal da Silva, T. J. Andersen, K. F. Jørgensen, A. Kudsk, 3D concrete printing of post-tensioned elements, Proceedings of IASS Symposium 2018-Creativity in Structural Design, Boston, July 16-20, 2018, 1-8.

[15] N. Roussel, A. Gram, M. Cremonesi, L. Ferrara, K. Krenzer, V. Mechtcherine, S. Shyshko, J. Skocek, J. Spangenberg, O. Svec, L. Thrane, K. Vasilic, Numerical simulations of concrete flow: A benchmark comparison, Cem Concr Res (2016) 79: 265-271. https://doi.org/10.1016/i.cemconres.2015.09.022

[16] N. Roussel, S. Staquet, L. D’Aloia Schwarzenstruber, R. Le Roy, F. Toutlemonde, SCC casting prediction for the realization of prototype VHPC-precambered composite beams, Mater Struct (2007) 40: 877887. https://doi.org/10.1617/s11527-006-9190-0

[17] J. Spangenberg, N. Roussel, J. H. Hattel, J. Thorborg, M. R. Geiker, H. Stang, J. Skocek, Prediction of the impact of flow induced inhomogeneities in self-compacting concrete (SCC), Proceedings of the 6th international RILEM symposium on SCC, (2010) 209-215.
[18] C.W. Hirt, B.D. Nichols, Volume of fluid (VOF) method for the dynamics of free boundaries, J Comput Phys (1981) 39: 201-225. https://doi.org/10.1016/0021-9991(81)90145-5.

[19] R. J. M. Wolfs, F. P. Bos, T. A. M. Salet, Hardened properties of 3D printed concrete: The influence of process parameters on interlayer adhesion. Cem Concr Res (2019) 119: 132-140. https://doi.org/10.1016/j.cemconres.2019.02.017 